\title{
Nominal Predication and Focus Anchoring ${ }^{*}$
}

\author{
Sze-Wing Tang \\ The Hong Kong Polytechnic University \\ sw.tang@polyu.edu.hk
}

\begin{abstract}
It will be shown that verbs can be missing in predicative sentences by using the data from Chinese. Copula-less sentences in Chinese are subject to 'Generalized Anchoring Principle' (GAP), which requires that every clause be anchored at the interface for LF convergence. To satisfy GAP, clauses may be either tensed or focused. It is shown that copula-less sentences in Chinese are subject to focus anchoring. It will be further argued that whether a verb is needed in predication depends on the syntax of predicate nominals.
\end{abstract}

\section{Introduction}

In English, every sentence must have a verb. Basically, sentences without a verb are ungrammatical.

(1) John *(is) a genius.

(2) John *(is) very clever.

Suppose that the existence of verbs is a mandatory requirement in English. Some questions arise:

(3) a. Is such a requirement universal?

b. If it is not universal, how can verbs be omitted?

c. If omission of verbs is permitted by Universal Grammar, why do we need verbs?

I will try to answer these three questions in sections 2,3 , and 4 , respectively. The focus of the discussion in this paper will be on Chinese copula-less predicative sentences.

\section{$2 \quad$ Nonexistence of verbs}

Chinese is a language that has verbs. For example, shi 'be' and xihuan 'like' are verbs in Chinese.
Ta shi Deguoren.
he be German
'He is a German.'

\footnotetext{
* I wish to express my sincere gratitude to a number of people for their input and constructive comments along the way as I have pursued the research in this paper, including Horst-Dieter Gasde, Ewald Lang, Paul Law, Thomas Lee, Susan Rothstein, Dingxu Shi, Wei-Tien Tsai, Chris Wilder, Ning Zhang, as well as participants in the Workshop on Predicative Constructions held at Zentrum für Allgemeine Sprachwissenschaft (ZAS), October 16-17, 2000. Needless to say, defects are not the fault of any of them. This work is partially supported by the Hong Kong Polytechnic University Central Research Grant G-S971 and funding from ZAS.
} 
Wo xihuan dongtian.

I like winter

'I like winter.'

The claim that every sentence needs a verb can be immediately refuted by Chinese as omission of verbs is allowed in Chinese. There are two major types of 'verbless' sentences in Chinese, namely 'copula-less sentences' and 'empty verb sentences'. Consider the data given below.

\section{Copula-less sentences}

(6) Jintian xingqiyi.

today Monday

'Today is Monday.'

(7) Zhangsan Zhongguoren.

Zhangsan Chinese

'Zhangsan is a Chinese.'

\section{Empty verb sentences}

(8) Wo niurou mian, Zhangsan zhurou mian.

I beef noodle Zhangsan pork noodle

'I order/eat beef noodle and Zhangsan pork noodle.'

(9) Meige ren san-ben shu.

every person three-Cl book

'Everyone has three books.'

A major difference between copula-less sentences and empty verb sentences is that the second nominal in copula-less sentences is a predicate while the second nominal in empty verb sentences is the logical object of the event. The relation between the two nominals in the copula-less sentences is 'predication': the second nominal is a predicate nominal which is predicated of the first nominal, i.e. the subject. ${ }^{2}$

Predicate nominals in the copula-less sentences denote the character and quality of the subject. They can be common nouns, proper names, and numerals.

Zhangsan [shagua].

(common nouns)

Zhangsan fool

'Zhangsan is a fool.'

(11) Zhege haizi [da yanjing].

this kid big eye

'This kid has big eyes.'

(12) Qu nian [huang nian], jin nian [feng nian].

last year famine year this year bumper year

'Last year was a famine year and this year a bumper year.'

(13) Wo [Zhangsan].

I Zhangsan

'I am Zhangsan.'

(proper names)

1 'Empty verb sentences' are also known as 'eventive constructions' in Zhang (2000).

${ }^{2}$ Other differences between these two types of verbless sentences are: (i) unlike the copula-less sentences, the second nominal in the empty verb sentences should not be existential/indefinite (Tang 1998, Zhang 2000), and (ii) the interpretation of the relation between the two nominals in the empty verb sentences relies on discourse information (Wang 2000). 
(14) Yi-bei kafei [wu kuaiqian].

(numerals)

one- $\mathrm{Cl}$ coffee five dollar

'A cup of coffee costs five dollars.'

(15) Ta [sanshi sui].

he thirty year

'He is thirty years ago.'

The copula shi 'be' can be inserted in most of the copula-less sentences. For example, both $(16)(=(7))$ and $(17)$ are acceptable in Chinese.

(16) Zhangsan Zhongguoren.

Zhangsan Chinese

'Zhangsan is a Chinese.'

(17) Zhangsan shi Zhongguoren.

Zhangsan be Chinese

'Zhangsan is a Chinese.'

Notice that omission of the copula $s h i$ 'be' is restricted to predicative sentences. The copula shi 'be' cannot be omitted in specificational sentences and equative sentences.

(18) Wo mai de *(shi) zhe duo hua.

I buy $\mathrm{DE}$ be this $\mathrm{Cl}$ flower

(specificational)

'What I bought is this flowers.'

[description - item]

(19) Zhe duo hua (shi) wo mai de.

this $\mathrm{Cl}$ flower be I buy DE

'This flower is what I bought.'

[item - description]

(20) Acht*(shi) ba.

eight be eight

(equative)

'Acht is eight.'

(21) $\mathrm{Ba} *$ (shi) acht.

eight be eight

'Eight is acht.'

Although some sentences are regarded as predicative sentences, the copula shi 'be' cannot be omitted. Based on the contrast among $(22)(=(10)),(23)$, and (24), we may assume that the copula shi 'be' can be omitted in predicative sentences only when the predicate nominal is not preceded by the numeral-classifier phrase. ${ }^{3}$

Zhangsan shagua.

Zhangsan fool

'Zhangsan is a fool.'

(23)

Zhangsan *(shi) yi-ge shagua.

Zhangsan be one-Cl fool

'Zhangsan is a fool.'

\footnotetext{
${ }^{3}$ Zhang (2000) argues that shagua 'fool' in (24) undergoes NP raising. Suppose that predicate nominals in Chinese copula-less sentences must be 'bare' (Tang 1998 and our discussion in section 4 of this paper). After NP raising, the predicate nominal becomes 'bare' in a sense that it is not c-commanded by the numeral-classifier phrase and thus (24) obeys the bareness requirement.
} 
Furthermore, the omission of the copula shi 'be' is prohibited in the 'coda' construction, such as $(25){ }^{4}$

Wu-li you yi-ge ren [*(shi) shagua].

(the 'coda' construction)

house-in have one-Cl person be fool

'There is a person in the room who is a fool.'

By using the data from Chinese, I have shown in this section that copula-less predicative sentences are grammatical in Chinese. Consequently, the claim that every sentence must have a verb should not be universal.

\title{
3 Constraints on copula-less predicative sentences
}

\subsection{Salvaging devices for making an unnatural copula-less predicative sentence natural}

Although copula-less predicative sentences exist in Chinese, their use is not unconstrained. In some situation, copula-less sentences may sound unnatural. For example, (26) and (27) are 'unnatural' and 'incomplete' if they are uttered in an out-of-the-blue context. In this subsection, I will illustrate how the judgment of copula-less sentences in Chinese can be improved.

\author{
?? Zhangsan xuesheng. \\ Zhangsan student \\ 'Zhangsan is a student.' \\ ?? Ta xiaotou. \\ he thief \\ 'He is a thief.'
}

First of all, juxtaposing a copula-less sentence with a parallel one in a contrast structure will improve the judgment. Compare (26) with (28).

Zhangsan xuesheng, Lisi jiaoshou.

Zhangsan student Lisi professor

'Zhangsan is a student and Lisi a professor.'

Second, modifying the predicate nominal by an adjective may also improve the judgment, particularly when an adjective that has an 'evaluative' judgment is inserted. For example, the predicate nominal in $(29 a)(=(26))$ is unmodified. The sentence becomes more natural once the adjective hao 'good' is inserted. There is a contrast between (29a) and (29b).

\footnotetext{
${ }^{4}$ To explain the ungrammaticality of (25), one possibility is to assume that the coda must be 'clausal' (Wilder 2000). If the bracketed element without shi 'be' in (25) is not a clause, it cannot be the coda. What happens if the coda without $s h i$ 'be' is a bare small clause, i.e. a clause without a verb? If Wilder (2000) is right, a null operator undergoes movement out of the coda. The ungrammaticality of (25) is due to extraction out of bare small clauses, which violates constraints on movement (Tang 1998).
} 
a. ?? Zhangsan xuesheng.

Zhangsan student

'Zhangsan is a student.'

b. Zhangsan hao xuesheng.

Zhangsan good student

'Zhangsan is a good student.'

Third, even if one does not add any adjective, if the meaning conveyed by the predicate nominal is 'specific' enough, we can produce sentences like (30b) and (31b) as 'natural' and 'complete' sentences. 'Specificity' here refers to a subset of a presupposed set. For example, daxue sheng 'university student' in (30b) is a subset of xuesheng 'student'. The former should be more 'specific' than the latter. In (31b), as the meaning of Zhongguoren 'Chinese' is more 'specific' than ren 'human', the judgment of (31b) is much better than (31a).
a. ?? Zhangsan xuesheng.
Zhangsan student
'Zhangsan is a student.'
b. Zhangsan daxue sheng.
Zhangsan university student
'Zhangsan is a university student.'
a. Z Zhangsan ren.
Zhangsan human
'Zhangsan is a human being.'
b. Zhangsan Zhongguoren.
Zhangsan Chinese
'Zhangsan is a Chinese.'

Fourth, it may be more felicitous if the predicate nominal conveys the speaker's judgment and attitude rather than fact. For example, the (b) sentences convey more subjective judgment of the speaker than those (a) sentences in (32) and (33). The copula-less sentences in (32b) and (33b) describe a characterization about which an opinion or judgment can be expressed.
a. ?? Zhangsan xuesheng.
Zhangsan student
'Zhangsan is a student.'
b. Zhangsan shagua.
Zhangsan fool
'Zhangsan is a fool.'
a. ?? Zhangsan nanren.
Zhangsan man
'Zhangsan is a man.'
b. Zhangsan nanzihan.
Zhangsan man
'Zhangsan is a man (more vivid).'

Fifth, adding a focus adverb, such as cai 'only', may improve the sentence, as in (34). However, notice that having other kinds of adverbs, such as temporal adverbs, does not help. 
For example, even though temporal adverbs jintian 'today' and gang 'just' are added in (35), the copula-less sentence still sounds 'unnatural' and 'incomplete'.

Zhangsan cai zhujiao.

Zhangsan only research assistant

'Zhangsan is only a research assistant.'

* Zhangsan jintian/gang zhujiao.

Zhangsan today/just research assistant

'Zhangsan is (just) a research assistant (today).'

Last but not least, I observe that embedding the copula-less sentence within a larger sentence may help complete the sentence." The embedded contexts encode "epistemic modality'. Copula-less sentences are not permitted in 'factual' contexts. See the contrast between (36) and (37).

$$
\begin{aligned}
& \text { Wo dang [Zhangsan xuesheng]. } \\
& \text { I consider Zhangsan student } \\
& \text { 'I consider Zhangsan a student.' } \\
& \text { * Wo zhidao [Zhangsan xuesheng]. } \\
& \text { I know Zhangsan student } \\
& \text { '*I know Zhangsan a student.' }
\end{aligned}
$$

Based on the above discussion, we may notice that the use of copula-less sentences is not unconstrained in Chinese. Their usage will be more natural only in some particular contexts. The contexts that may contribute to the 'naturalness' and 'completeness' of copulaless sentences in Chinese can be summarized in (38).

\section{(38) Contexts that contribute to naturalness of copula-less sentences in Chinese}

(a) in contrastive contexts

(b) having a modified predicate nominal

(c) having a 'specific' predicate nominal

(d) having a subjective judgment

(e) having a focus adverb

(f) embedding

Without the above contexts, copula-less sentences become 'unnatural' and 'incomplete'. If these contexts are regarded as 'salvaging devices', it seems that copula-less sentences can be 'licensed' by any one of these devices in order to be used naturally and freely. Are these contexts unrelated? Do they share any similarities? Can we further derive any generalizations from these contexts listed in (38)? I will address all these questions in the next subsection.

\subsection{Generalized Anchoring Principle}

Before discussing the properties of copula-less sentences in Chinese, let me spell out a working hypothesis in this paper. I assume that all sentences, including copula-less sentences in Chinese, are subject to a constraint that requires that every sentence in natural language be licensed at the interface levels, which is dubbed as 'Generalized Anchoring Principle' or 'GAP' (Tang and Lee 2000).

\footnotetext{
${ }^{5}$ The bracketed constituent in (36) is also known as a 'small clause'. See Tang (1998) for a detailed discussion on the small clause construction in Chinese.
} 
Every clause must be either tensed or focused at the LF interface level.

Why do we need GAP? Even though a derivation that derives a linguistic expression violates no principles internal to the computation system of human language $\mathrm{C}_{\mathrm{HL}}$, such as economy principles and cyclicity, the expression is not necessarily ready to be used. Universal Grammar has to make sure that every object generated by $\mathrm{C}_{\mathrm{HL}}$ is accommodated to the external systems.

Along these lines, GAP, which requires that every sentence be anchored, is imposed at the $\mathrm{LF}$ interface from the external systems that make use of the information provided by $\mathrm{C}_{\mathrm{HL}}$. In other words, GAP can be regarded as a 'bare output condition'. In this vein, the examples of copula-less sentences I have shown in the previous subsection are considered to be incomplete because they are not anchored in order to be fully interpretable at the LF interface and to be used by the external systems.

There are two strategies to satisfy GAP in natural languages: sentences are either tensed or focused in the sense that it highlights an item in contrast to a set of alternatives supplied by the context of utterance.

In the case of tense, on a par with the analysis of tense by Enç (1987), an event is anchored with respect to the moment of speech or a reference event. In the case of focus, I propose that an item is anchored with respect to a reference set of items, or an event is anchored vis-à-vis a reference set of events.

'Focus' discussed in this paper refers to the inducing of a contrasting set of individuals, properties or events by means of focusing devices associating with constituents in a sentence, a notion central to most theories of focus (cf. Konig 1991, Krifka 1992, Rooth 1992). It subsumes phenomena such as 'symmetric contrastive focus' explored in Rooth (1992), which involves two clauses or sentences, or even a single sentence in which there are two elements of the same type in focus; one contrasting with the other. Anchoring by focus provides another route to temporal anchoring, satisfying GAP. Chinese?

How does GAP account for the salvaging devices for copula-less sentences in

Contrast structures such as those in (28) make it clear that we are speaking of an arrangement of participants and situations having a 'list reading'. The copula-less sentence is juxtaposed with an alternative situation. The invoking of a contrast set is a key element underlying focus structure.

Regarding the role of the adjectives in the modified predicate nominals, they may introduce new information in certain contexts and receive a contrastive stress or contrastive accent. For example, the adjective hao 'good' in (29b) can be marked a new, as repeated in (40a). With intonation focus, (40a) contributes a set of propositions of the form, such as (40b) to the representation, which can be regarded as a set of alternatives to the assertion ' $\mathrm{He}$ is a good student' in the sense of Rooth (1992).

\footnotetext{
a. Zhangsan HAO xuesheng. Zhangsan good student 'Zhangsan is a GOOD student.'

b. Zhangsan is a $x$ student.
} 
I assume that the 'specific' predicate nominals and those predicate nominals that convey a subjective judgment of the speaker have a similar effect as what the modified predicate nominals have. They are contrasted with some presupposed properties. For example, in $(30 \mathrm{~b})$, as repeated in (41), the speaker seems to contrast the predicate nominal with other properties: 'Zhangsan is a UNIVERSITY student (and not an ordinary student).' In (32b), as repeated in (42), the predicate nominal shagua 'fool' is highlighted in contrast to a set of alternatives: 'Zhangsan is a FOOL (and not a genius).'

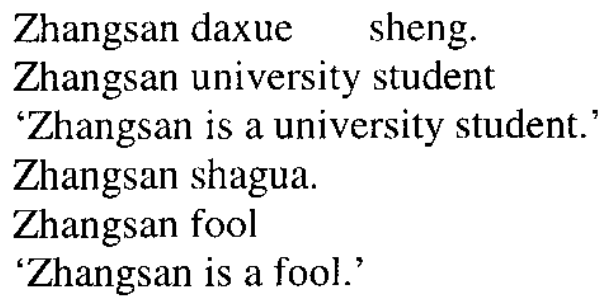

The observation that the predicate nominal in copula-less sentences is juxtaposed with some presupposed properties seems to be reminiscent of Ma's (1998) analysis of Chinese predicate nominals. According to him, a nominal that can be used as a predicate in Chinese should convey an 'ordinal meaning'. For example, chuntian 'spring', xiatian 'summer', qiutian 'fall', and dongtian 'winter' are in a particular sequence and their meaning is known as the 'ordinal meaning'.

Along these lines, the nominal daxue sheng 'university student' in (41) can be associated with other nominals, such as xiaoxue sheng 'elementary school student' and zhongxue sheng 'high school student', all of which are in a sequence and convey an 'ordinal meaning'. Hence, the acceptability of (41) is predicted under Ma's (1998) analysis.

However, as noted by Ma (1998:67), acceptable examples like (31b) and (42) are problematic in his analysis as some Chinese nominals that denote properties, such as Zhongguoren 'Chinese' in (31b) and shagua 'fool' in (42), have nothing to do with the 'ordinal meaning'. Their acceptability will be unexplained unless we propose some ad hoc definitions of ordinality (cf. Ma 1998:68 fn 9).

Without appealing to any ad hoc solutions, focus anchoring provides a unified and very natural explanation: all these contexts induce focusing effects in contrasting the situation depicted with an alternative set of situations. The copula-less sentences with modified predicate nominals and those having a 'specific' meaning and a subjective judgment are all anchored by focus, satisfying GAP.

In the case of having a focus adverb in copula-less sentences, such as cai 'only' in (34), the focus adverb invokes a contrast set and induces focusing effects in contrasting the situation depicted with an alternative set of situations.

If a copula-less sentence is embedded in a context that denotes epistemic modality, the matrix epistemic verb, such as dang 'consider' in (36), contributes to focus anchoring in that modality in embedding contrasts a possible world with the actual state of affairs.

Focus anchoring may save some apparently unacceptable copula-less sentences in Chinese. As noted by Shi (2001), it is normally unacceptable in isolation if the predicate nominal is too long, such as (43). He points out that the judgment will be improved if a 'welldefined' context is provided to force a particular reading. For example, (43) will be acceptable if it is given as the answer to a question like (44). Under the present analysis, we may say that the 'well-defined' contexts for copula-less sentences are those anchored by focus, satisfying GAP. If the long predicate nominal Jiaodong bandao toushang yi-ge xiao yu-cun-de ren 'a 
person from a small fishing village at the tip of the Jiaodong Peninsula' in (43) is contrasted with Liaodong bandao ren 'people from the Liaodong Peninsula' in (44), the copula-less sentence (43) will be anchored by focus and thus it becomes acceptable. ${ }^{6}$

(??) Wo [Jiaodong bandao toushang yi-ge xiao yu-cun-de ren]. I Jiaodong peninsula tip one-Cl small fishing-village-Mod person 'I am a person from a small fishing village at the tip of the Jiaodong Peninsula.' Women dou shi Liaodong bandao ren. $\mathrm{Ni}$ ne? we all be Liaodong peninsula person you $\mathrm{Q}$ 'We are all from the Liaodong Peninsula. What about you?'

If the discussion in this paper is on the right track, copula-less sentences in Chinese will become 'natural' and 'complete' unless they are anchored. All the unnaturalness of the copula-less sentences is due to the violation of GAP. The so-called salvaging devices are all subsumed under focus anchoring.

\section{Copula-less sentences in Chinese should be anchored by focus.}

If (45) is a correct generalization for Chinese, is it a language-particular rule? Why is it the case that copula-less predicative sentences are not easily found in English? How is the parametric variation between Chinese and English with respect to predicative sentences accounted for? All these questions will be addressed in the next section.

\section{Syntax of copula-less sentences}

Recall that in section 2 I have argued that the claim that every sentence must have a verb is not universal. For example, the copula can be omitted in predicative sentences in Chinese. If omission of the copula is possible in natural language, why can't verbs be omitted in English predicative sentences?

I propose that nominals in natural languages can be classified into two types: 'predicative' and 'non-predicative'. Predicative nominals vs. non-predicative nominals can be defined in a sense of Higginbotham's (1985) $\theta$-binding: a predicative nominal has an open place in it, which has to be closed off by a referential category whereas the open place in a non-predicative nominal is closed off (see also Stowell 1991). In terms of syntax, all NP nominals are basically predicative. If the nominals are dominated by a functional projection, for instance Determiner Phrase DP, they are non-predicative or 'argumental' (Szabolcsi 1987, 1992, Stowell 1991a,b, Longobardi 1994). Predicative nominals and non-predicative nominals may serve as predicates and arguments, respectively.

\section{Predicative nominals: e.g. NP}

Non-predicative nominals: e.g. DP

Let us assume that predicative nominals can be predicated of the subject directly without any verbal categories whereas non-predicative nominals can't. Consider the contrast between (47a) and (47b), in which 'SU' stands for the subject. ${ }^{7}$ In (47a), N (or N') is

\footnotetext{
${ }^{6}$ Meanwhile copula-less sentences in Chinese are perhaps constrained by some discourse factors, in addition to focus anchoring. See Shi (2001) for a discussion along these lines.

${ }^{7}$ (47a) should be permitted by Universal Grammar. See Stowell (1983).
} 
predicative as it is not headed by any functional categories. On the other hand, N (or NP) in (47b) is headed by D and thus is no longer predicative. The element in the specifier of DP cannot serve as the subject for the NP. The configuration in (47b) is ungrammatical.

a.

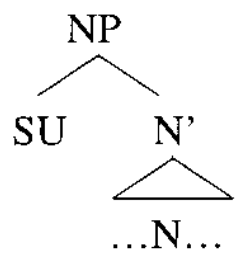

b.

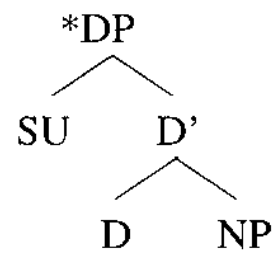

Now let us consider some empirical data. In (48) a genius is a DP whose head is realized as the article $a$ (Abney 1987). As a genius is not predicative, it cannot be predicated of the subject John directly without a verb. (48) will have a structure like (47b) and should be ungrammatical.

* John a genius.

In order to make predication possible in (48), a verbal category is needed in the structure. However, bare verbs are prohibited in English, as illustrated in (49). Whenever there is a verb in English, it must be associated with some tense morphology. The contrast between (49) and (50) shows that the copula in English predicative sentences is inflected to indicate tense. Along these lines, verbs cannot be missing in English as they are required to support the inflectional suffixes. I assume that the copula be in English is used to bear tense features; its existence is required by tense.

(49) * John be a genius.

(50) John is/was a genius.

Even if we suppose that there is a 'null verb' in English, its existence is ruled out as it cannot support the inflectional tense morphemes. For example, the inflectional morphemes are supposed to be attached to a 'null verb' in (51). (51) is ruled out by the morphology of English that suffixes cannot be attached to null elements.

* John -s/-ed a genius.

In any event, (48) is ungrammatical in English regardless of whether a contrast set is invoked to anchor (48), such as (52). If (48) is already ruled out by syntax, focus anchoring does not help.

(52) * John a genius, and Bill an idiot.

The situation in Chinese copula-less sentences is different. The predicate nominal shagua 'fool' in $(53)(=(42))$ is a bare noun. As it is a bare noun, it can be predicated of the subject Zhangsan directly, having a structure similar to $(47 \mathrm{a}) .^{8}$

\footnotetext{
${ }^{8}$ Shi (2000) argues that the first nominal in copula-less sentences should be a 'subject' rather than a 'topic'.
} 
The discussion here has a very interesting implication: the existence of copula-less predicative sentences is associated with the syntax of the predicate nominal. If the predicate nominal is 'bare' in English, it is predicated that it may enter copula-less sentences. I notice that in some contexts, verbs can be missing in predicative sentences in English and the prediction is borne out. Consider the following examples.

(55) You Martha, me professor.

(56) Next station Jordan.

Expressions like (54) are known as 'you idiot expressions', which are mainly used in exclamations involving a strong value judgment and an opinion (Tang 1998). Unless being a student is associated with some bad quality and having a negative status, (57) may not sound natural. The contrast between (54) and (57) suggests that only the nominals that have an 'evaluative meaning' may felicitously enter the you idiot expressions.

\# You student!

(55) is recorded from a conversation in a movie. What the speaker of (55) wanted to convey was to emphasize the contrast of the identity between the hearer and himself.

(56) is from the broadcast in Mass Transit Railway in Hong Kong. Similar expressions can also be found in German. For example, (58) is from the broadcast in S-Bahn in Berlin. Interestingly, the copula is always missing in such expressions.

Nächste Bahnhof Friedrichstraße.

next station Friedrichstraße

In (54) and (55), as the predicate nominals idiot and professor do not have any determiner and article, they are regarded as 'bare' and are not dominated by DP. Bare NPs are predicative and they can be predicated of the subject directly. On the other hand, if there is a determiner, such as (59), the judgment is deviant. The ungrammaticality of (59) is obvious: the predicate nominal an idiot is a non-predicative DP by virtue of the existence of the article and thus it cannot be predicated of the subject directly.

\section{* You an idiot!}

Although Martha in (55) and Jordan in (56) are proper names, they are used as indefinite common nouns in those two sentences. Such an indefinite usage of proper names is not impossible in natural languages. For example, plural markers can be attached to proper names, expressing an indefinite meaning in English $(=(60))$ as well as in Chinese $(=(61))$. I assume that definite proper names are in the D position (Abney 1987) while indefinite proper names are Ns (Longobardi 1994, Li 1999).

(60) I saw three Johns this morning. 
Zhangsan-men shenme shihou lai?

Zhangsan-Pl what time come

'When are Zhangsans (or Zhangsan and the others) coming?'

How are the copula-less sentences in (54)-(56) in common? (54) has a strong evaluative context. Although such a strong evaluative meaning is not involved in the locative expressions, the predicate nominal, such as Jordan in (56), is contrasted with some presupposed stations. The hearer is expected to be aware that Jordan is one of the stations along the railroad. Similar to some copula-less sentences in Chinese (e.g. (28)), (55) is a contrast structure and thus it is licensed by focus anchoring. In other words, all these copulaless sentences in English are anchored by focus, satisfying GAP. It seems that the generalization stated in (45) can also hold in English.

Data from Chinese and English show that copula-less predicative sentences are anchored by focus only. Why is it the case that these sentences are anchored by focus instead of tense?

I propose that the choice of GAP is determined by syntax. Suppose that there must be a tense operator in temporal anchoring. Enç (1987) argues that tense is indexical like all other referential expressions and is conceived of as a pronominal variable, in that the truth of a tensed sentence is relative to the speech time. ${ }^{9}$ A tense is anchored through its complementizer $\mathrm{C}$ (or a tense operator embedded in $\mathrm{CP}$ ).

As copula-less predicative sentences are bare, there is no $\mathrm{CP}$ in the structure and thus the tense operator cannot occur. Temporal anchoring is never available in copula-less sentences. ${ }^{10}$ If the discussion here is on the right track, the generalization stated in (45) that copula-less sentences in Chinese is only anchored by focus can be derived from the syntax of copula-less predicative sentences.

The bareness property of copula-less predicative sentences can be extended to English. The accusative Case of the subject $m e$ in (55) may support the present analysis that copulaless predicative sentences are bare. Let us assume with Schïtze (1997) that the 'default' Case of the English subject is the accusative Case when it cannot get the nominative Case. If the copula-less predicative sentences in (55) are bare, the subject cannot receive the nominative Case from a functional category, for instance, $T$. Hence, the subject gets the default accusative Case. Focus anchoring in English copula-less predicative sentences follows the bareness of the structure in syntax.

\section{Conclusion}

In the literature, it is claimed that every sentence must have a verb in English. In the beginning of this paper, three questions regarding this claim were raised, as repeated in (62).

a. Is such a requirement universal?

b. If it is not universal, how can verbs be omitted?

c. If omission of verbs is permitted by Universal Grammar, why do we need verbs?

\footnotetext{
9 See also Partee (1973), Guéron and Hoekstra (1995), and Stowell (1996) for similar ideas.

${ }^{10}$ If we need a focus operator in focus anchoring, on a par with temporal anchoring, it could be the case that the focus operator can be adjoined to bare projections freely, regardless of whether there is CP.
} 
Regarding the first question, the answer is 'no'. I have shown that verbs can be missing in some Chinese sentences. Copula-less predicative sentences are possible in Chinese.

If the existence of verbs is not obligatory in some sentences, how can verbs be omitted? By using the data from Chinese, I have shown that copula-less predicative sentences are acceptable when they are (i) in contrastive contexts, (ii) having a modified predicate nominal, (iii) having a 'specific' predicate nominal, (iv) having a subjective judgment, (v) having a focus adverb, and (vi) embedded.

Regarding (62c), if omission of verbs is permitted by Universal Grammar, we may wonder why English needs a copula in predicative sentences in the first place. I proposed that the existence of copula-less predicative sentences is associated with the syntactic status of predicate nominals. Bare NP nominals are predicative whereas DP nominals are nonpredicative. Ungrammaticality of copula-less sentences in English is due to the morphology of tense as well as the usage of non-predicative predicate nominals. It is argued that if the predicate nominal is bare, the copula can be omitted. Supporting evidence comes from Chinese copula-less sentences, you idiot expressions, and locative expression in English. I have also argued that the unavailability of temporal anchoring in copula-less sentences is due to syntax.

\section{References}

Abney, Steven Paul (1987): The English Noun Phrase in its Sentential Aspect. Doctoral dissertation, MIT.

Enç, Mürvet (1991): The Semantics of Specificity. Linguistic Inquiry 22: 1-25.

Guéron, Jacqueline / Teun Hoekstra (1995): The Temporal Interpretation of Predication. In Anna Cardinaletti and Maria T. Guasti (eds.) Syntax and semantics 28, 77-107. San Diego: Academic Press.

Higginbotham, James (1985): On Semantics. Linguistic Inquiry 16, 547-593.

Konig, Ekkehard (1991): The Meaning of Focus Particles. London: Routledge.

Krifka, Manfred (1992): A Framework for Focus-Sensitive Quantification. In C. Barker and D. Dowty (eds.) Proceedings of the Second Conference on Semantics and Linguistic Theory. Columbus, Ohio: Department of Linguistics, Ohio State University.

Li, Y.-H. Audrey (1999): Plurality in a Classifier Language. Journal of East Asian Linguistics 8, 75-99.

Longobardi, Giuseppe (1994): Reference and Proper Names: a Theory of N-Movement in Syntax and Logical Form. Linguistic Inquiry 25, 609-665.

Ma, Qingzhu (1998): Hanyu Yuyi Yufa Fanchou Wenti [On Semantic Grammatical Categories in Chinese]. Beijing: Beijing Language and Culture University Press.

Partee, Barbara H. (1973): Some Structural Analogies between Tenses and Pronouns in English. The Journal of Philosophy 70: 601-609.

Rooth, Mats (1992): A Theory of Focus Interpretation. Natural Language Semantics 1: 75-116.

Schütze, Carson T. (1997): Infl in Child and Adult Language: Agreement, Case and Licensing. Doctoral dissertation, MIT.

Shi, Dingxu (2000): Topic and Topic-Comment Constructions in Mandarin Chinese. Language 76, 383-408.

Shi, Dingxu (2001): The Nature of Chinese Nominal Predicates. Ms., The Hong Kong Polytechnic University.

Stowell, Tim (1983): Subjects Across Categories. The Linguistic Review 2: 285-312.

Stowell, Tim (1991): Small Clause Restructuring. In Robert Freidin (ed.) Principles and Parameters in Comparative Grammar, 182-218. Cambridge, Mass.: The MIT Press.

Stowell, Tim (1996): The Phrase Structure of Tense. In Johan Rooryck and Laurie Zaring (eds.) Phrase Structure and the Lexicon, 271-286. Dordrecht: Kluwer Academic Publishers.

Szabolcsi, Anna (1987): Functional Categories in the Noun Phrase. In Approaches to Hungarian 2, $167-189$. Szeged: JATE.

Szabolcsi, Anna (1992): Subordination: Articles and Complementizers. In Approaches to Hungarian 4: the structure of Hungarian, ed. I Kenesei and Cs. Pléh, 123-137. Szeged: JATE.

Tang, Sze-Wing (1998): Parametrization of Features in Syntax. Doctoral dissertation, University of California, Irvine.

Tang, Sze-Wing / Thomas H.-T. Lee (2000): Focus as an Anchoring Condition. Paper presented at International Symposium on Topic and Focus in Chinese, The Hong Kong Polytechnic University.

Wang, Jie (2000): Zailun 'Shenglue Shuyu Dongci' de Tici Weiyuju [Revisit Substantive Predicate Sentences with an Omitted Verbal Predicate|. Ms., Nanjing University. 
Wilder, Christopher (2000): Syntax der Prädikation. Project proposal, ZAS, Berlin.

Zhang, Ning (2000): On Chinese Verbless Constructions. In Kerstin Schwabe and Ning Zhang (eds.) Ellipsis in Conjunction, 157-177. Tübingen: Max Niemeyer Verlag. 